\title{
Acessibilidade aos serviços odontológicos no SUS: revisão da literatura
}

\author{
Accessibility to dental services in SUS: a literature review \\ Accesibilidad a los servicios dentales em el SUS: revisión de la literatura
}

Kairo Silvestre Meneses Damasceno

ORCID: https://orcid.org/0000-0002-2444-4496 Universidade do Estado da Bahia, Brasil E-mail: kairodamasceno@hotmail.com

Denise Nogueira Cruz

ORCID: https://orcid.org/0000-0002-9523-9561 Universidade Federal da Bahia, Brasil

E-mail: dncnogueira@hotmail.com

Sandra Garrido de Barros

ORCID: https://orcid.org/0000-0002-8255-1230 Universidade Federal da Bahia, Brasil E-mail:sgb@ufba.br

\begin{abstract}
Resumo
Introdução: A saúde como um direito inerente à cidadania na Constituição de 1988 foi uma conquista de grupos sociais que, à época, insatisfeitos com as desigualdades e exclusão de parte da população aos serviços de saúde, lutaram pela consolidação do Sistema Único de Saúde (SUS). Através desta conquista, políticas públicas foram implementadas para garantir este direito. No entanto, as desigualdades de acesso aos serviços de saúde são, ainda, um problema presente que impede o efetivo exercício do SUS em virtude de barreiras encontradas em diferentes dimensões, como a estrutural, operacional e relacional. Objetivo: Revisar a produção científica sobre a acessibilidade aos serviços odontológicos do SUS, caracterizando as principais barreiras de acesso. Método: Revisão integrativa da literatura com busca ativa de artigos publicados no período de 2010 a 2017 nas bases de dados Scientific Electronic Library Online (SciELO) e Literatura Latino-Americana e do Caribe em Ciências da Saúde (LILACS), utilizando os descritores "acesso", "acessibilidade", "serviços odontológicos" e "saúde bucal". Resultados: Um total de 24 artigos foram selecionados, apresentando como principais barreiras de acesso a falta de informação sobre os serviços e da importância da saúde bucal, a autoavaliação quanto à saúde bucal, a falta de materiais, recursos humanos e insumos, a não efetivação das políticas públicas vigentes, dentre outros. Conclusão: A superação das barreiras de acesso é fundamental para diminuir as iniquidades sociais e promover a justiça social, bem como novos estudos voltados para os grupos considerados mais vulneráveis.
\end{abstract}

Palavras-chave: Acesso; Acessibilidade; Serviços odontológicos; Saúde bucal.

\begin{abstract}
Introduction: Health as an inherent right to citizenship in the 1988 constitution of Brazil was a conquest by social groups who, at the time, dissatisfied with inequalities and exclusion of part of the population to health services, fought to consolidate the Unified Health System (SUS). Through this conquest, public policies were implemented to guarantee this right. However, unequal access to health services is still a problem which hinders the effective exercise of SUS due to the barriers found in various aspects, such as structural, operational and relational. Objective: Review of scientific production on accessibility to SUS dental services was performed, characterizing the main barriers to access. Method: Integrative literature review with search of articles published between 2010 and 2017 was carried out on the Scientific Electronic Library Online (SciELO) and Latin American and Caribbean literature on Health Sciences (LILACS) databases, using the following keywords: "access", "accessibility", "dental services" and "oral health". Results: A total of 24 articles were selected, presenting as main barriers to access the lack of information about services and the importance of oral health, self-assessment of oral health, the lack of materials, human resources and supplies, the failure to implement current public policies, among others. Conclusion: Overcoming these barriers to access is fundamental in order to diminish social inequities and to promote social justice, as well as new studies aimed at the groups considered most vulnerable.
\end{abstract}

Keywords: Access; Accessibility; Dental services; Oral health.

\section{Resumen}

Introducción: La salud como derecho inherente a la ciudadanía en la Constitución de 1988 fue un logro de grupos sociales que, en su momento, estaban insatisfechos con las desigualdades y la exclusion de parte de la población a los servicios de salud, lucharon por la consolidación del Sistema Único de Salud (SUS). A través de este logro, se 
implementaron políticas públicas para garantizar esto derecho. Sin embargo, las desigualdades en el acceso a los servicios de salud son, aún así, un problema presente que impiede el ejercício efectivo del SUS debido a barreras encontradas en diferentes dimensiones, tales como estructurales, operacionales y relacional. Objetivo: Revisar la producción científica sobre el accesibilidad a los servicios dentales del SUS, caracterizando las principals barreras de acceso. Método: Revisión de la literatura con búsqueda activa de artículos publicados de 2010 a 2017 en las bases de datos Scientific Electronic Library Online (SciELO) y Literatura Latinoamericana y Caribeña sobre Ciencias de la Salud (LILACS), utilizando las palabras clave "acceso", “accesibilidad", "servicios dentales" y "salud dental". Resultados: Um total de 24 artículos fueron seleccionados, presentando como principales barreras de acesso la falta de información sobre los servicios y la importância de la salud bucal, autoevaluación de la salud bucal, la falta de materiales, recursos humanos e insumos, la falta de implementación de las políticas públicas vigentes, entre otros. Conclusión: Superar estas barreras de acceso es fundamental para reducir inequidades sociales y promover la justicia social, así como nuevos estudios dirigidos a los grupos considerados más vulnerables.

Palabras clave: Acceso; Accesibilidad; Servicios dentales; Salud bucal.

\section{Introdução}

A Constituição de 1988 conferiu à saúde o status de direito social, próprio à condição de cidadão, cabendo ao poder público garanti-lo. A concepção do Sistema Único de Saúde (SUS), portanto, está vinculada a ideia de que todas as pessoas têm direito à saúde. Pauta-se no conceito de seguridade social e admite uma sociedade orientada na igualdade e na equidade, sem discriminação ou privilégios (Paim, 2016). Programas e políticas públicas de saúde como o Programa Saúde da Família (PSF), a Política Nacional de Atenção Básica (PNAB) e a Política Nacional de Saúde Bucal (PNSB) têm sido formulados no sentido de aproximarem os serviços públicos de saúde aos princípios e diretrizes do SUS.

O Ministério da Saúde (MS) lançou, em 1994, o Programa Saúde da Família (PSF). A partir de 2006, este programa configurou-se como estratégia prioritária para a reorganização da atenção básica no país, passando a ser denominada Estratégia de Saúde da Família (ESF). A PNAB contribuiu para a expansão da atenção básica de forma a desenvolver uma atenção integral à saúde, desenvolver a autonomia dos sujeitos e estimular a participação social (Brasil, 2006; Barros, Medina, Carcereri, Ruiz, \& Peres, 2015).

Em 28 de dezembro de 2000, a Portaria nº 1444 criou e regulamentou o incentivo financeiro para a inclusão da Equipe de Saúde Bucal na ESF, com o objetivo de melhorar os índices epidemiológicos e ampliar o acesso da população aos serviços de saúde bucal. A partir deste momento, os investimentos nessa área foram crescentes (Brasil, 2000; Barros, Medina, Carcereri, Ruiz, \& Peres, 2015). A inclusão da Saúde Bucal na ESF garantiu a ampliação do acesso à saúde bucal e da integralidade dos serviços ofertados na atenção primária, superando a prática mutiladora das exodontias, bem como o acesso à informação em saúde bucal e sobre os serviços ofertados (Fonseca, Nehmy \& Mota, 2015). Permitiu, ainda, a articulação com as propostas de vigilância à saúde e com o conceito de cuidado integral, tendo como foco as famílias (Brasil, 2004; Mattos, Ferreira, Leite, \& Greco, 2014; Fonseca, Nehmy \& Mota, 2015).

Nesse sentido, a Política Nacional de Saúde Bucal (PNSB), de 2004, ampliou a assistência odontológica da população com práticas voltadas para a promoção, prevenção e tratamento, de maneira a reorganizar os serviços e reorientar o modelo de atenção à saúde, fortalecendo a Estratégia de Saúde da Família (ESF) e ampliando o acesso aos serviços especializados (Brasil, 2004).

O significado de "acesso" remete à ideia de "utilização" do serviço de saúde para a satisfação das necessidades em saúde. O direito ao acesso à saúde legitimado pela Constituição como exercício da cidadania, controle social e equidade, o torna um importante dispositivo de transformação da realidade (Travassos \& Martins, 2004; Abreu-de-Jesus \& Assis, 2010; Fonseca, Fonseca \& Meneghim, 2017).

Os fatores relacionados ao acesso aos serviços de saúde conformam modelos explicativos a exemplo do modelo proposto por Andersen (1995), discutido em estudos sobre o tema, em que os fatores de predisposição consistem nas características demográficas, como gênero e idade; na estrutura social, como a escolaridade; e nas crenças e conhecimento da 
existência dos serviços. Já os fatores de capacidade permitem ou impedem a utilização dos serviços de saúde, como seguro de saúde, renda e distância geográfica. A influência da necessidade de saúde, por sua vez, diz respeito às necessidades percebidas ou diagnosticadas, sendo relacionadas com a autopercepção (Viegas, Carmo \& Luz, 2015; Cruz, Chaves \& Cangussu, 2016).

Uma das formas de se medir o acesso aos serviços de saúde é através da sua utilização, sendo que a literatura aponta desigualdades neste padrão com associações entre nível econômico, onde a baixa renda constitui-se uma barreira de acesso; faixa etária, com maior utilização dos serviços pelas mulheres e pelos adultos jovens; nível de escolaridade, em que os mais escolarizados têm maior acesso (Souza \& Chaves, 2010; Rocha-Buelvas, 2013; Cruz, Chaves \& Cangussu, 2016).

Quanto ao termo acessibilidade, Travassos e Martins (2004) abordam a concepção de Donabedian (1973, 2003) que se refere aos fatores que facilitam (ou não) a utilização dos serviços de saúde pela população e que dependem das dimensões geográficas e sócio-organizacionais. Os fatores geográficos caracterizam-se pela distância percorrida pelos usuários até os serviços de saúde, pela topografia que pode apresentar escadarias, subidas e descidas, pelo custo e pelo tempo de locomoção. Já os fatores sócio governamentais relacionam-se com as características organizacionais e de oferta dos serviços, como as formas de triagem, marcação de consultas e fluxo. Há, ainda, fatores sociais, culturais e religiosos que também influenciam na acessibilidade aos serviços de saúde.

A realização desta pesquisa justifica-se pela necessidade de se discutir as dificuldades de acesso aos serviços de saúde bucal por parte da população, identificando, na literatura, as principais lacunas e limitações relacionadas ao tema de forma a reorientar ações que permitam garantir direitos do cidadão, princípios e diretrizes do SUS. Outrossim, a pesquisa possibilita fomentar reflexões acerca do tema, gerando conhecimentos que possam contribuir com estado da arte.

Desta forma, este trabalho objetiva revisar a produção científica sobre a acessibilidade aos serviços odontológicos do SUS, com o intuito de sistematizar e analisar os principais fatores que facilitam/dificultam o acesso e as estratégias de enfrentamento às barreiras de acesso aos serviços odontológicos.

\section{Metodologia}

Foi realizada uma revisão integrativa da literatura por meio de pesquisa de artigos nas bases de dados eletrônicas Scientific Electronic Library Online (SciELO) e Literatura Latino-Americana e do Caribe em Ciências da Saúde (LILACS) nos anos de 2010 a 2017. A revisão integrativa constitui um método de pesquisa que sintetiza, de forma sistemática, os resultados do conhecimento acerca de um determinado tema investigado, podendo contribuir, no contexto da saúde, para intervenções na assistência e tomadas de decisão (Mendes, Silveira \& Galvão, 2008).

Os artigos foram selecionados a partir dos seguintes descritores: "acesso", "acessibilidade", "serviços odontológicos" e "saúde bucal". Foi utilizado o operador booleano lógico "AND" com pareamento dos seguintes termos: ("acesso" AND "saúde bucal") e ("acessibilidade" AND "serviços odontológicos"). Também foram aplicados os filtros: texto completo, idiomas português, inglês e espanhol e tipo de documento "artigo completo". Na SciELO, a busca foi realizada considerandose como campo de busca "todos os índices" e na LILACS; as "palavras".

A pesquisa foi realizada nos meses de fevereiro de 2018 a abril de 2018, tendo, como critérios de exclusão, artigos publicados anteriormente ao ano de 2010, aqueles que não se enquadravam no tema acessibilidade à saúde bucal, além de publicações referentes a resumos de congressos, anais, editoriais e trabalhos de conclusão de curso. Do total de 24 artigos selecionados, 12 foram encontrados na base de dados LILACS através dos descritores "acesso" e "saúde bucal" e 07 foram selecionados na base de dados SciELO usando os mesmos descritores; 04 artigos foram selecionados na base de dados LILACS e 01 na base SciELO com os descritores "acessibilidade" e "serviços odontológicos", conforme Quadro 1. Os artigos que apresentaram duplicidade nas bases de dados foram contabilizados na base na qual foi primeiro encontrado. 
Para fins de seleção dos artigos incluídos, foram considerados os fatores que influenciam no acesso propostos por Andersen (1995), como os fatores de predisposição, de capacitação e de necessidade de saúde.

Quadro 1: Síntese da busca e seleção de artigos científicos, segundo descritores utilizados e bases de dados selecionadas.

\begin{tabular}{|c|c|c|c|}
\hline Descritores & ETAPAS & SciELO & LILACS \\
\hline \multirow[t]{3}{*}{$\begin{array}{l}\text { "Acesso" AND "saúde } \\
\text { bucal" }\end{array}$} & $\begin{array}{l}\text { Busca nos anos de } \\
2010 \text { e } 2017\end{array}$ & 193 & 326 \\
\hline & $\begin{array}{c}\text { Leitura de título e } \\
\text { relação com o tema de } \\
\text { estudo }\end{array}$ & 20 & 36 \\
\hline & $\begin{array}{l}\text { Leitura de resumos e } \\
\text { exclusão por } \\
\text { duplicidade }\end{array}$ & 07 & 12 \\
\hline \multicolumn{4}{|c|}{ Total de 19 artigos com os descritores "acesso" AND "saúde bucal" } \\
\hline \multirow[t]{3}{*}{$\begin{array}{l}\text { "Acessibilidade" AND } \\
\text { "serviços odontológicos" }\end{array}$} & $\begin{array}{c}\text { Busca nos anos de } \\
2010 \text { e } 2017\end{array}$ & 01 & 51 \\
\hline & $\begin{array}{l}\text { Leitura de título e } \\
\text { relação com o tema de } \\
\text { estudo }\end{array}$ & 01 & 13 \\
\hline & $\begin{array}{c}\text { Leitura de resumos e } \\
\text { exclusão por } \\
\text { duplicidade }\end{array}$ & 01 & 04 \\
\hline \multicolumn{4}{|c|}{ Total de 05 artigos com os descritores "acessibilidade" AND "serviços odontológicos" } \\
\hline \multicolumn{4}{|c|}{ Total de 24 artigos selecionados } \\
\hline
\end{tabular}

Fonte: Autores.

Foram selecionados, ao final, 24 artigos, sendo 19 provenientes da SciELO e 05 da LILACS, após o uso dos filtros e critérios de exclusão. A relação dos 24 artigos selecionados e respectivas informações sobre base de dados em que foram localizados, título, autoria, periódico, ano de publicação e tipo de pesquisa encontra-se no Quadro 2.

Quadro 2: Artigos selecionados a partir da revisão de literatura sobre a acessibilidade aos serviços públicos odontológicos segundo base de dados e ordem cronológica de publicação.

\begin{tabular}{|c|c|c|c|c|}
\hline $\begin{array}{c}\text { Base de } \\
\text { dados }\end{array}$ & TÍTULO & AUTOR (ano) & Periódico & Tipo de artigo \\
\hline \multirow[t]{5}{*}{ LILACS } & $\begin{array}{l}\text { Acessibilidade dos idosos brasileiros } \\
\text { aos serviços odontológicos. }\end{array}$ & $\begin{array}{l}\text { Viana, Gomes, } \\
\text { Carvalho \& } \\
\text { Oliveira }(2010)\end{array}$ & $\begin{array}{l}\text { Revista da } \\
\text { Faculdade de } \\
\text { Odontologia } \\
\end{array}$ & Revisão \\
\hline & $\begin{array}{l}\text { Política nacional de saúde bucal: } \\
\text { acessibilidade e utilização de serviços } \\
\text { odontológicos especializados em um } \\
\text { município de médio porte na Bahia. }\end{array}$ & $\begin{array}{l}\text { Souza \& Chaves } \\
(2010)\end{array}$ & $\begin{array}{l}\text { Revista Baiana } \\
\text { Saúde Pública }\end{array}$ & empírico \\
\hline & $\begin{array}{l}\text { Acessibilidade ao Serviço de Saúde } \\
\text { Bucal na Atenção Básica: } \\
\text { Desvelando o Absenteísmo em uma } \\
\text { Unidade de Saúde da Família de João } \\
\text { Pessoa-PB. }\end{array}$ & $\begin{array}{l}\text { Melo, Braga \& } \\
\text { Forte (2011) }\end{array}$ & $\begin{array}{l}\text { Revista Brasileira } \\
\text { de Ciências da } \\
\text { Saúde }\end{array}$ & empírico \\
\hline & $\begin{array}{l}\text { Access to dental services and related } \\
\text { factors in adolescents from Vitória, } \\
\text { Espírito Santo, Brazil, } 2011 .\end{array}$ & $\begin{array}{l}\text { Emerich et al. } \\
\text { (2011) }\end{array}$ & $\begin{array}{l}\text { Brazilian Research } \\
\text { in Pediatric } \\
\text { Dentistry and } \\
\text { Integrated Clinic }\end{array}$ & empírico \\
\hline & $\begin{array}{l}\text { Estudo da acessibilidade } \\
\text { organizacional aos serviços de saúde }\end{array}$ & $\begin{array}{l}\text { Castro, Oliveira \& } \\
\text { Araújo (2011) }\end{array}$ & $\begin{array}{l}\text { Revista Brasileira } \\
\text { de Ciências da }\end{array}$ & empírico \\
\hline
\end{tabular}




\begin{tabular}{|c|c|c|c|}
\hline $\begin{array}{l}\text { bucal de um município de pequeno } \\
\text { porte do nordeste brasileiro. }\end{array}$ & & Saúde & \\
\hline $\begin{array}{l}\text { Acesso à assistência odontológica no } \\
\text { acompanhamento pré-natal. }\end{array}$ & $\begin{array}{l}\text { Neto, Oliveira, } \\
\text { Zandonade \& Leal. } \\
\text { (2012) }\end{array}$ & $\begin{array}{l}\text { Ciência \& Saúde } \\
\text { Coletiva }\end{array}$ & empírico \\
\hline $\begin{array}{l}\text { Utilização e Acesso aos Serviços de } \\
\text { Saúde Bucal do SUS -Uma } \\
\text { Comparação entre Populações Rurais } \\
\text { e Urbanas. }\end{array}$ & $\begin{array}{l}\text { Cavalcanti, Gaspar } \\
\text { \& Goes(2012) }\end{array}$ & $\begin{array}{l}\text { Pesquisa Brasileira } \\
\text { em Odontopediatria } \\
\text { Clínica e Integrada }\end{array}$ & empírico \\
\hline $\begin{array}{l}\text { Características do acesso e utilização } \\
\text { de serviços odontológicos em } \\
\text { municípios de médio porte }\end{array}$ & $\begin{array}{l}\text { Chaves et al. } \\
(2012)\end{array}$ & $\begin{array}{l}\text { Ciência \& Saúde } \\
\text { Coletiva }\end{array}$ & empírico \\
\hline $\begin{array}{l}\text { Análisis sobre el acceso a los } \\
\text { servicios de la salud bucal: um } \\
\text { indicador de equidad. }\end{array}$ & $\begin{array}{l}\text { Rocha-Buelvas } \\
\text { (2013) }\end{array}$ & $\begin{array}{l}\text { Revista Gerencia y } \\
\text { Políticas de Salud }\end{array}$ & Revisão \\
\hline $\begin{array}{l}\text { Desigualdade na utilização de } \\
\text { serviços de saúde bucal na atenção } \\
\text { básica e fatores associados em dois } \\
\text { municípios brasileiros. }\end{array}$ & $\begin{array}{l}\text { Soares, Chaves \& } \\
\text { Cangussu (2013) }\end{array}$ & $\begin{array}{l}\text { Revista } \\
\text { Panamericana de } \\
\text { Salud Pública }\end{array}$ & empírico \\
\hline $\begin{array}{l}\text { A organização do acesso aos serviços } \\
\text { de saúde bucal na estratégia de saúde } \\
\text { da família de um município da Bahia. }\end{array}$ & $\begin{array}{l}\text { Tavares, Costa, } \\
\text { Falcão \& Cristino. } \\
(2013)\end{array}$ & Saúde em Debate & empírico \\
\hline $\begin{array}{l}\text { Melhoria do acesso à saúde bucal na } \\
\text { Estratégia de Saúde da Família: uma } \\
\text { revisão de literatura. }\end{array}$ & $\begin{array}{l}\text { Sanchez \& Braga. } \\
(2014)\end{array}$ & $\begin{array}{l}\text { Revista de Atenção } \\
\text { Primária à Saúde }\end{array}$ & Revisão \\
\hline $\begin{array}{l}\text { Avaliação da acessibilidade ao } \\
\text { Centro de Especialidades } \\
\text { Odontológicas de abrangência } \\
\text { macrorregional } \\
\text { de Sobral, Ceará, Brasil. } \\
\end{array}$ & Pinto et al. (2014) & $\begin{array}{l}\text { Ciência \& Saúde } \\
\text { Coletiva }\end{array}$ & empírico \\
\hline $\begin{array}{l}\text { O valor social dos dentes e o acesso } \\
\text { aos serviços odontológicos. }\end{array}$ & $\begin{array}{l}\text { Fonseca, Nehmy \& } \\
\text { Mota. (2015) }\end{array}$ & $\begin{array}{l}\text { Ciência \& Saúde } \\
\text { Coletiva }\end{array}$ & empírico \\
\hline $\begin{array}{l}\text { Análise do acesso aos serviços } \\
\text { odontológicos públicos no Brasil. }\end{array}$ & $\begin{array}{l}\text { Fonseca, Fonseca } \\
\& \text { Meneghim. } \\
(2017)\end{array}$ & $\begin{array}{l}\text { ABCS Health } \\
\text { Sciences }\end{array}$ & Revisão \\
\hline $\begin{array}{l}\text { Utilização de serviços de saúde bucal } \\
\text { na atenção primária: perspectivas dos } \\
\text { usuários do SUS de um município do } \\
\text { sul do Brasil. }\end{array}$ & Solano et al. (2017) & $\begin{array}{l}\text { Revista Ciência } \\
\text { Plural }\end{array}$ & empírico \\
\hline $\begin{array}{l}\text { Inequalities in access and utilization } \\
\text { of dental services: a cross-sectional } \\
\text { study in an área covered by the } \\
\text { Family Health Strategy. }\end{array}$ & $\begin{array}{l}\text { Baldani \& Antunes } \\
\text { (2011) }\end{array}$ & $\begin{array}{l}\text { Caderno de Saúde } \\
\text { Pública }\end{array}$ & empírico \\
\hline $\begin{array}{l}\text { Uso regular de serviços } \\
\text { odontológicos entre adultos e idosos } \\
\text { em região vulnerável no sul do } \\
\text { Brasil. }\end{array}$ & $\begin{array}{l}\text { Machado, } \\
\text { Camargo, } \\
\text { Jeronymo \& } \\
\text { Bastos. (2012) }\end{array}$ & $\begin{array}{l}\text { Revista de Saúde } \\
\text { Publica }\end{array}$ & empírico \\
\hline $\begin{array}{l}\text { Uso de serviços odontológicos entre } \\
\text { pré-escolares: } \\
\text { estudo de base populacional. }\end{array}$ & $\begin{array}{l}\text { Rodrigues et al. } \\
(2014)\end{array}$ & $\begin{array}{l}\text { Ciência \& Saúde } \\
\text { Coletiva }\end{array}$ & empírico \\
\hline $\begin{array}{l}\text { Acessibilidade a serviços de saúde } \\
\text { bucal por pessoas idosas: uma revisão } \\
\text { integrativa. }\end{array}$ & $\begin{array}{l}\text { Austregésilo, Leal, } \\
\text { Marques, Vieira \& } \\
\text { Alencar (2015) }\end{array}$ & $\begin{array}{l}\text { Revista Brasileira } \\
\text { de Geriatria e } \\
\text { Gerontologia } \\
\end{array}$ & revisão \\
\hline $\begin{array}{l}\text { Utilização dos serviços odontológicos } \\
\text { por pré-escolares em um } \\
\text { município de pequeno porte do } \\
\text { Estado da Paraíba. }\end{array}$ & $\begin{array}{l}\text { Paredes, } \\
\text { Fernandes, } \\
\text { Fernandes \& } \\
\text { Menezes (2015) }\end{array}$ & $\begin{array}{l}\text { Revista de } \\
\text { Odontologia da } \\
\text { UNESP }\end{array}$ & empírico \\
\hline $\begin{array}{l}\text { As dimensões do acesso aos serviços } \\
\text { de saúde bucal na mídia impressa. }\end{array}$ & $\begin{array}{l}\text { Esposti, Cavaca, } \\
\text { Côco, Santos-Neto } \\
\text { \& Oliveira (2016) }\end{array}$ & Saúde e Sociedade & revisão \\
\hline
\end{tabular}


Research, Society and Development, v. 10, n. 3, e17610313194, 2021

(CC BY 4.0) | ISSN 2525-3409 | DOI: http://dx.doi.org/10.33448/rsd-v10i3.13194

\begin{tabular}{|l|l|l|l|l|}
\hline & $\begin{array}{l}\text { Equidade no uso de serviços } \\
\text { odontológicos provenientes do SUS } \\
\text { entre idosos: estudo de base } \\
\text { populacional. }\end{array}$ & $\begin{array}{l}\text { Oliveira, Souza, } \\
\text { Haikal, Ferreira \& } \\
\text { Martins (2016) }\end{array}$ & $\begin{array}{l}\text { Ciência \& Saúde } \\
\text { Coletiva }\end{array}$ & empírico \\
\cline { 2 - 4 } & $\begin{array}{l}\text { Uso de serviços odontológicos de } \\
\text { forma regular } \\
\text { na população de Montes Claros, MG, } \\
\text { Brasil. }\end{array}$ & $\begin{array}{l}\text { Carreiro et al. } \\
(2017)\end{array}$ & $\begin{array}{l}\text { Ciência \& Saúde } \\
\text { Coletiva }\end{array}$ & empírico \\
\hline
\end{tabular}

Fonte: Autores.

\section{Resultados e Discussão}

Dos 24 artigos selecionados, uma pequena proporção $(16,7 \%)$ abordava o acesso aos serviços odontológicos pela população idosa (Viana, Gomes, Carvalho \& Oliveira, 2010; Machado, Camargo, Jeronymo \& Bastos, 2012; Austregésilo, Leal, Marques, Vieira \& Alencar, 2015; Oliveira, Souza, Haikal, Ferreira \& Martins, 2016;), sendo que um destes incluiu, também, a população adulta (Machado, Camargo, Jeronymo \& Bastos, 2012). Entre a produção selecionada, um artigo (4,17\%) estudou o acesso por adolescentes (Emerich et al., 2015); dois (8,3\%) por pré-escolares (Paredes, Fernandes, Fernandes \& Menezes, 2015; Rodrigues et al., 2014) e um (4,17\%) relacionou o pré-natal de gestantes com a acessibilidade aos serviços odontológicos (Neto, Oliveira, Zandonade \& Leal, 2012).

A maior parcela $(37,5 \%)$ foi de artigos que reportavam a acessibilidade aos serviços odontológicos em regiões ou munícipios, seja através de estudo local em unidades básicas de saúde (Baldani \& Antunes, 2011; Castro, Oliveira \& Araújo, 2011; Melo, Braga \& Forte, 2011; Carreiro et al., 2017; Solano et al., 2017; Tavares, Costa, Falcão \& Cristino, 2013) ou comparativos entre municípios ou entre área urbana e rural de um mesmo município (Cavalcanti, Gaspar \& Goes, 2012; Chaves et al., 2012; Soares, Chaves \& Cangussu, 2013). Dois artigos (8,3\%) analisaram o acesso aos serviços odontológicos especializados (Souza \& Chaves, 2010; Pinto et al., 2014) e cinco (20,8\%) abordaram o tema de forma mais generalizada, sem direcionamento para um grupo ou região específica (Rocha-Buelvas, 2013; Sanchez \& Braga, 2014; Fonseca, Nehmy \& Mota, 2015; Esposti, Cavaca, Côco, Santos-Neto \& Oliveira, 2016; Fonseca, Fonseca \& Meneghim, 2017). Observou-se que, em todos os anos do período analisado, houve artigos publicados sobre a acessibilidade aos serviços odontológicos.

Apesar da produção selecionada reportar as vulnerabilidades como um óbice ao acesso aos serviços de saúde bucal, não foram encontrados estudos referentes às populações negra e de Lésbicas, Gays, Bissexuais, Travestis, Transexuais ou Transgêneros, Queer, Intersexo, Assexual e mais (LGBTQIA+), às comunidades indígenas e ribeirinhas, nem ao grupo etário de homens adultos que, apesar de não serem considerados vulneráveis, usam menos os serviços. Quanto a questão de gênero, Machado, Camargo, Jeronymo e Bastos (2012) relatam que as mulheres utilizam 30\% a mais os serviços odontológicos quando comparadas aos homens. Além disso, não foram encontrados estudos que aprofundassem a questão do acesso dos homens aos serviços de saúde bucal e possíveis estratégias para alcançá-los.

O sistema incremental de atendimento odontológico voltado a uma população específica, com foco em escolares e em trabalhadores formais, excluía boa parte da população à assistência odontológica; limitação esta amenizada pela implantação da Estratégia Saúde da Família e das Equipes de Saúde Bucal (Narvai, 2006; Paim, 2016). Portanto, mensurar o acesso aos serviços de saúde é fundamental para o planejamento de ações e de políticas de saúde que contribuam para o uso e melhoria destes serviços (Andersen \& Davidson, 2000).

Os principais fatores associados ao acesso à saúde bucal e à utilização dos serviços odontológicos identificados nos artigos revisados foram: sexo (Souza \& Chaves, 2010; Melo, Braga \& Forte, 2011; Castro, Oliveira \& Araújo, 2011; Baldani \& Antunes, 2011; Chaves et al., 2012; Machado, Camargo, Jeronymo \& Bastos, 2012; Soares, Chaves \& Cangussu, 2013; Solano et al., 2017), idade (Cavalcanti, Gaspar \& Goes, 2012; Austregésilo, Leal, Marques, Vieira \& Alencar, 2012; 
Machado,Camargo, Jeronymo \& Bastos, 2012; Rocha-Buelvas, 2013; Rodrigues et al., 2014; Paredes, Fernandes, Fernandes \& Menezes, 2015), renda (Souza \& Chaves, 2010; Castro, Oliveira \& Araújo, 2011; Melo, Braga \& Forte, 2011; Chaves et al., 2012; Machado, Camargo, Jeronymo \& Bastos, 2012; Paredes, Fernandes, Fernandes \& Menezes, 2015; Oliveira, Souza, Haikal, Ferreira \& Martins, 2016), localização geográfica (Pinto et al., 2010; Souza \& Chaves, 2010; Cavalcanti, Gaspar \& Goes, 2012; Rocha-Buelvas, 2013; Carreiro et al., 2017), falta de insumos e manutenção de equipamentos (Castro, Oliveira \& Araújo, 2011; Tavares, Costa, Falcão \& Cristino, 2013), tempo de espera (Souza \& Chaves, 2010; Castro, Oliveira \& Araújo, 2011; Melo, Braga \& Forte, 2011; Chaves et al. 2012), acesso à informação de saúde (Neto, Oliveira, Zandonade \& Leal, 2012; Austregésilo, Leal, Marques, Vieira \& Alencar, 2015; Fonseca, Fonseca \& Meneghim, 2017; Solano et al., 2017) e autopercepção de saúde bucal (Viana, Gomes, Carvalho \& Oliveira, 2010; Baldani \& Antunes, 2011; Castro, Oliveira \& Araújo, 2011; Melo, Braga \& Forte, 2011; Machado, Camargo, Jeronymo \& Bastos, 2012; Rodrigues et al., 2014; Austregésilo, Leal, Marques, Vieira \& Alencar, 2015; Emerich et al., 2015; Fonseca, Nehmy \& Mota, 2015; Oliveira, Souza, Haikal, Ferreira \& Martins, 2016; Carreiro et al., 2017; Fonseca, Fonseca \& Meneghim, 2017).

Rocha-Buelvas (2013) aborda a equidade em saúde como a ausência de diferenças injustas ao acesso a bens e serviços de saúde. Neste contexto, a identificação de grupos vulneráveis aos riscos, que poderiam não estar acessando adequadamente os serviços sanitários, seria um aspecto fundamental na abordagem científica e política das iniquidades em saúde. Nesta direção, as estratégias para enfrentamento das barreiras de acesso e iniquidades em saúde encontradas nos artigos selecionados foram relacionadas no Quadro 3.

Quadro 3: Fatores que determinam ou dificultam o acesso aos serviços públicos odontológicos e as estratégias para enfrentamento das iniquidades de acesso da saúde bucal de acordo com os artigos selecionados.

\begin{tabular}{|c|c|c|}
\hline $\begin{array}{l}\text { AUTORES } \\
\text { (ano) }\end{array}$ & $\begin{array}{c}\text { Principais fatores que determinam / } \\
\text { dificultam o acesso }\end{array}$ & Estratégias \\
\hline $\begin{array}{l}\text { Viana, Gomes, } \\
\text { Carvalho \& } \\
\text { Oliveira }(2010)\end{array}$ & $\begin{array}{l}\text { Limitações econômicas, sociodemográficas, } \\
\text { culturais, percepção da necessidade. }\end{array}$ & $\begin{array}{l}\text { Superar as iniquidades sociais e a escassez da oferta dos serviços } \\
\text { odontológicos. }\end{array}$ \\
\hline $\begin{array}{l}\text { Souza \&Chaves } \\
(2010)\end{array}$ & $\begin{array}{l}\text { Barreiras organizacionais: tempo para } \\
\text { realização da consulta, barreiras geográficas: } \\
\text { tempo de deslocamento. Sexo, renda. }\end{array}$ & $\begin{array}{l}\text { Unidades básicas de saúde como locais para agendamento para o } \\
\text { Centro de Especialidade Odontológica (CEO), lista diária de } \\
\text { espera para substituição dos faltosos, adequação da carga horária } \\
\text { dos profissionais, localização do CEO em áreas mais centrais, } \\
\text { consulta com hora marcada. }\end{array}$ \\
\hline $\begin{array}{l}\text { Melo, Braga \& } \\
\text { Forte, }(2011)\end{array}$ & $\begin{array}{l}\text { Tempo de espera para consulta elevado, acesso } \\
\text { às informações, menor renda, baixa } \\
\text { escolaridade, autopercepção da saúde bucal, } \\
\text { sexo, idade. }\end{array}$ & $\begin{array}{l}\text { Promover condições adequadas de atendimento, sistema de } \\
\text { agendamento que diminua o tempo de espera, } \\
\text { corresponsabilização dos usuários quanto ao cuidado com a } \\
\text { saúde. }\end{array}$ \\
\hline $\begin{array}{l}\text { Emerich et al. } \\
\text { (2011) }\end{array}$ & $\begin{array}{l}\text { Boa cobertura da estratégia de saúde da família } \\
(100 \%) \text { na região estudada, boa percepção de } \\
\text { saúde. }\end{array}$ & $\begin{array}{l}\text { Alta cobertura da estratégia saúde da família e percepção } \\
\text { positiva dos adolescentes em relação à saúde oral. }\end{array}$ \\
\hline $\begin{array}{l}\text { Castro, Oliveira \& } \\
\text { Araújo (2011) }\end{array}$ & $\begin{array}{l}\text { Falta de medicamentos, falta de material ou } \\
\text { equipamentos quebrados, longa espera para } \\
\text { consulta, existência de filas, dificuldade de } \\
\text { agendamento, percepção de saúde, sexo, renda. }\end{array}$ & $\begin{array}{l}\text { Ações planejadas de forma participativa, implantação das } \\
\text { políticas públicas de saúde bucal, capacitação dos profissionais } \\
\text { de saúde. }\end{array}$ \\
\hline $\begin{array}{l}\text { Neto, Oliveira, } \\
\text { Zandonade \& Leal } \\
(2012)\end{array}$ & $\begin{array}{l}\text { Poucas informações específicas da odontologia } \\
\text { às gestantes. }\end{array}$ & $\begin{array}{l}\text { Educação em saúde como superação das barreiras de acesso, } \\
\text { articulação de políticas de saúde mais específicas. }\end{array}$ \\
\hline $\begin{array}{l}\text { Cavalcanti, Gaspar } \\
\& \text { Goes (2012) }\end{array}$ & Idade e zona de residência do indivíduo. & Implementação das políticas e organização dos serviços. \\
\hline $\begin{array}{l}\text { Chaves et al. } \\
(2012)\end{array}$ & $\begin{array}{l}\text { Não retorno da ficha de contrarreferência à } \\
\text { atenção básica, tempo elevado para consulta de } \\
\text { retorno, sexo, renda. }\end{array}$ & $\begin{array}{l}\text { Marcação permanente de consultas, substituição de faltosos e } \\
\text { eliminação de filas, estímulo à continuidade do cuidado, } \\
\text { fortalecimento da capacidade gerencial local. }\end{array}$ \\
\hline $\begin{array}{l}\text { Rocha-Buelvas } \\
\text { (2013) }\end{array}$ & $\begin{array}{l}\text { Questões econômicas como principais barreiras } \\
\text { de acesso aos serviços odontológicos. }\end{array}$ & $\begin{array}{l}\text { Identificação das barreiras de acesso e dos grupos } \\
\text { potencialmente vulneráveis como abordagem das iniquidades em } \\
\text { saúde. }\end{array}$ \\
\hline Soares, Chaves \& & escolaridade, & Ações preventivas específicas, educação em saúde. \\
\hline
\end{tabular}




\begin{tabular}{|c|c|c|}
\hline Cangussu (2013) & socioeconômicos. & \\
\hline $\begin{array}{l}\text { Tavares, Costa, } \\
\text { Falcão \& Cristino } \\
(2013)\end{array}$ & $\begin{array}{l}\text { Falta de materiais, deficiência na manutenção } \\
\text { de equipamentos, baixa cobertura do serviço. }\end{array}$ & $\begin{array}{l}\text { Ampliação da cobertura, identificação dos grupos vulneráveis, } \\
\text { participação social. }\end{array}$ \\
\hline $\begin{array}{l}\text { Sanchez \& Braga } \\
(2014)\end{array}$ & $\begin{array}{l}\text { Contextos políticos e organizacionais dos } \\
\text { municípios, profissionais sem perfil adequado, } \\
\text { não integração das equipes de saúde bucal na } \\
\text { Estratégia Saúde da Família (ESF). }\end{array}$ & $\begin{array}{l}\text { Práticas de saúde voltadas para a integralidade, escuta } \\
\text { qualificada, trabalho em equipe, vigilância em saúde e } \\
\text { intersetorialidade. }\end{array}$ \\
\hline Pinto et al. (2014) & $\begin{array}{l}\text { Distância, falta de transportes, barreiras } \\
\text { financeiras, como o custo com transporte e } \\
\text { barreiras organizacionais, como a carência de } \\
\text { oferta no terceiro turno. }\end{array}$ & $\begin{array}{l}\text { Ampliar a oferta do serviço especializado, promover } \\
\text { oportunidades de utilização dos serviços. }\end{array}$ \\
\hline $\begin{array}{l}\text { Fonseca, Nehmy } \\
\& \text { Mota }(2015)\end{array}$ & $\begin{array}{l}\text { Autopercepção da saúde bucal, falta de acesso a } \\
\text { informações }\end{array}$ & $\begin{array}{l}\text { Reconhecimento da importância simbólica dos dentes e legitimar } \\
\text { o acesso à reabilitação odontológica como parte do direito à } \\
\text { saúde. }\end{array}$ \\
\hline $\begin{array}{l}\text { Fonseca, Fonseca } \\
\& \text { Meneghim } \\
\text { (2017) }\end{array}$ & $\begin{array}{l}\text { Determinantes socioeconômicos, demográficos } \\
\text { e de serviços. }\end{array}$ & $\begin{array}{l}\text { Redução das desigualdades socioeconômicas, ações } \\
\text { prevenção e de controle social. }\end{array}$ \\
\hline $\begin{array}{l}\text { Oliveira, Souza, } \\
\text { Haikal, Ferreira \& } \\
\text { Martins (2016) }\end{array}$ & $\begin{array}{l}\text { Aspectos sociodemográficos, acesso a } \\
\text { informações em saúde. }\end{array}$ & Implementação das políticas públicas existentes. \\
\hline $\begin{array}{l}\text { Solano et al. } \\
(2017)\end{array}$ & $\begin{array}{l}\text { Falta de acesso à informação sobre a } \\
\text { disponibilidade dos serviços de saúde bucal nas } \\
\text { unidades, sexo, idade, escolaridade. }\end{array}$ & $\begin{array}{l}\text { Investir em infraestrutura, como sinalização interna das } \\
\text { unidades, acolhimento e educação permanente. }\end{array}$ \\
\hline $\begin{array}{l}\text { hi \& } \\
\text { es }(2011)\end{array}$ & $\begin{array}{l}\text { Autopercepção de saúde bucal, dificuldades de } \\
\text { conseguir uma vaga ou marcar uma consulta, } \\
\text { sexo, idade. }\end{array}$ & $\begin{array}{l}\text { Promover ações que ampliem o acesso para a população de baixa } \\
\text { renda. }\end{array}$ \\
\hline $\begin{array}{l}\text { Machado, } \\
\text { Camargo, } \\
\text { Jeronymo \& } \\
\text { Bastos (2012) }\end{array}$ & $\begin{array}{l}\text { Autopercepção de saúde bucal, nível de } \\
\text { escolaridade, sexo, renda. }\end{array}$ & $\begin{array}{l}\text { Ações que promovam o conhecimento } \\
\text { sobre a saúde bucal e melhoria do autocuidado, acesso a serviços } \\
\text { odontológicos voltados a integralidade da atenção. }\end{array}$ \\
\hline $\begin{array}{l}\text { Rodrigues et al. } \\
\text { (2014) }\end{array}$ & $\begin{array}{l}\text { Baixa condição socioeconômica, acesso a } \\
\text { informações, idade, autopercepção de saúde } \\
\text { bucal. }\end{array}$ & $\begin{array}{l}\text { Necessidade de oferta dos serviços e implementação de políticas } \\
\text { de saúde. }\end{array}$ \\
\hline $\begin{array}{l}\text { Austregésilo, Leal, } \\
\text { Marques, Vieria \& } \\
\text { Alencar (2015) }\end{array}$ & $\begin{array}{l}\text { Aspectos culturais, econômicos, } \\
\text { sociodemográficos, percepção da necessidade. }\end{array}$ & $\begin{array}{l}\text { Superar as iniquidades sociais e a escassez da oferta de serviços, } \\
\text { informação à população sobre a importância dos serviços } \\
\text { odontológicos. }\end{array}$ \\
\hline $\begin{array}{l}\text { Paredes, } \\
\text { Fernandes, } \\
\text { Fernandes \& } \\
\text { Menezes }(2015)\end{array}$ & $\begin{array}{l}\text { Acesso à informação, falta de ficha, demora / } \\
\text { espera, percepção de necessidade, idade, } \\
\text { aspectos sociodemográficos. }\end{array}$ & $\begin{array}{l}\text { Implementação de políticas públicas de saúde para o incentivo à } \\
\text { procura de atendimento odontológico, orientação aos pais e } \\
\text { responsáveis quanto à importância do uso dos serviços em idade } \\
\text { precoce. }\end{array}$ \\
\hline $\begin{array}{l}\text { Esposti, Cavaca, } \\
\text { Côco, Santos- } \\
\text { Neto, Oliveira } \\
(2016)\end{array}$ & $\begin{array}{l}\text { Oferta dos serviços odontológicos insuficientes; } \\
\text { fatores como renda e escolaridade, mídia } \\
\text { apresenta o SUS como um serviço público e } \\
\text { não como um bem público. }\end{array}$ & $\begin{array}{l}\text { Importância do papel da mídia em informar sobre a } \\
\text { disponibilidade e a qualidade do serviço odontológico no SUS. }\end{array}$ \\
\hline $\begin{array}{l}\text { Carreiro et al. } \\
(2017)\end{array}$ & $\begin{array}{lll}\text { Aspectos relacionados } & \text { ao } & \text { serviço, } \\
\text { autopercepção, idade. } & & \end{array}$ & $\begin{array}{l}\text { Melhoria das políticas públicas para o uso de serviços } \\
\text { odontológicos e monitoramento das condições de saúde bucal da } \\
\text { população. }\end{array}$ \\
\hline
\end{tabular}

Fonte: Autores.

Quanto ao sexo, a literatura analisada revela que as mulheres correspondem a maior porcentagem de usuários dos serviços de saúde bucal no SUS (Souza \& Chaves, 2010; Melo, Braga \& Forte, 2011; Castro, Oliveira \& Araújo, 2011; Baldani \& Antunes, 2011; Chaves et al., 2012; Machado, Camargo, Jeronymo \& Bastos, 2012; Soares, Chaves \& Cangussu, 2013; Solano et al. 2017). Os homens, além de utilizarem menos os serviços, são mais afetados por enfermidades como o câncer bucal, atribuído a uma maior exposição a fatores de risco como o alcoolismo e o tabagismo (Petersen, Bourgeois, Ogawa, Estupinan-Day \& Ndiaye, 2005; Santos, Freitas, Andrade \& Oliveira, 2010). A associação do gênero feminino à maior utilização dos serviços de saúde pode ser explicada pelo fato de as mulheres possuírem maior preocupação com a saúde e autocuidado (Machado, Camargo, Jeronymo \& Bastos, 2012). 
O acesso aos serviços odontológicos varia com a idade do indivíduo, sendo que crianças na idade de 5 anos correspondem a maior porcentagem dos indivíduos que nunca foram ao dentista (Cavalcanti, Gaspar \& Goes, 2012; Rodrigues et al, 2014; Paredes, Fernandes, Fernandes \& Menezes, 2015).

Entre os pais/responsáveis que nunca levaram suas crianças ao dentista, a falta de interesse foi o principal motivo relatado, podendo ser influenciada pela ausência de conhecimento sobre a importância da dentição decídua e dos cuidados de saúde bucal em crianças. A educação em saúde seria, portanto, uma forma de enfrentamento a esta barreira ao acesso de préescolares. Entre os pais que haviam levado seus filhos ao dentista, os fatores que dificultaram o atendimento à criança foram demora para o atendimento, ausência de profissional, falta de tempo do responsável pela criança, distância e o fato de a criança não ter cooperado durante a consulta (Paredes, Fernandes, Fernandes \& Menezes, 2015).

Foi observada que a prevalência do uso dos serviços odontológicos é inversamente proporcional ao aumento da idade (Viana, Gomes, Carvalho \& Oliveira, 2010; Machado, Camargo, Jeronymo \& Bastos, 2012; Austregésilo, Leal, Marques, Vieira \& Alencar, 2015), ou seja, menor entre os idosos, podendo ser explicado pela perda de dentes e não percepção quanto a outros cuidados orais; pela dificuldade de acesso aos serviços odontológicos e pelas condições socioeconômicas (Viana, Gomes, Carvalho \& Oliveira, 2010). Um estudo, entretanto, identificou a utilização dos serviços odontológicos do SUS maior entre idosos em estado de vulnerabilidade (Oliveira, Souza, Haikal, Ferreira \& Martins, 2016).

Outras abordagens, na busca de explicações para baixa utilização dos serviços odontológicos entre idosos, destacam que, nesta fase da vida, a atenção está voltada para problemas sistêmicos, aliado ao desconhecimento da relação das condições bucais com estes (Viana, Gomes, Carvalho \& Oliveira, 2010). Assim, a idade pode ser um importante fator de determinação da menor frequência de visitas ao dentista pelos idosos.

As iniquidades em saúde e os fatores socioeconômicos refletem no uso dos serviços odontológicos e nas condições de saúde bucal da população, de forma que, quanto maior a renda, melhor o acesso ou utilização dos serviços. Isso demonstra as dificuldades do SUS em garantir o acesso universal e a equidade (Peres et al., 2012; Esposti, Cavaca, Côco, Santos-Neto \& Oliveira, 2016). Assim, a baixa renda constitui um fator limitador de acesso à saúde bucal, sendo observada em alguns estudos como perfil da maioria da população estudada (Souza \& Chaves, 2010; Viana, Gomes, Carvalho \& Oliveira, 2010; Machado, Camargo, Jeronymo \& Bastos 2012; Austregésilo, Leal, Marques, Vieira \& Alencar, 2015; Oliveira, Souza, Haikal, Ferreira \& Martins, 2016; Carreiro et al., 2017).

Dentre os fatores que dificultam o acesso e a utilização dos serviços de saúde estão as condições socioeconômicas desfavoráveis (Rocha-Buelvas, 2013; Austregésilo, Leal, Marques, Vieira \& Alencar, 2015). Para populações idosas e economicamente desfavorecidas, por exemplo, a renda, muitas vezes, é utilizada para o sustento de outros membros da família, sendo, portanto, uma limitação econômica para a utilização dos serviços odontológicos (Viana, Gomes, Carvalho \& Oliveira, 2010). Todavia, um estudo revela que o uso dos serviços odontológicos no SUS foi maior entre os idosos em estado de vulnerabilidade e com condições econômicas mais desfavoráveis, o que sugere que a busca pelo princípio da equidade está presente (Oliveira, Souza, Haikal, Ferreira \& Martins, 2016).

As principais barreiras geográficas relacionaram-se a grandes distâncias a percorrer do domicílio até a unidade de saúde (Pinto et al., 2010; Cavalcanti, Gaspar \& Goes, 2012), tempo gasto de deslocamento (Souza \& Chaves, 2010; Carreiro et al., 2017) e dificuldade de mobilidade (Cavalcanti, Gaspar \& Goes, 2012; Rocha-Buelvas, 2013). Essas barreiras se tornam mais evidentes em zonas rurais em razão da grande distância até os centros urbanos, de estradas sem manutenção, precariedade de transporte público, dentre outros (Hobdell, 2007; Cavalcanti, Gaspar \& Goes, 2012).

Souza e Chaves (2010) avaliaram o tempo de deslocamento até o serviço odontológico especializado como um obstáculo percebido: 46,6\% dos usuários levaram mais de 40 minutos de deslocamento, enquanto para 44,6\% esse tempo variou de 16 a 40 minutos, sendo que o tempo médio ideal de deslocamento seria de 15 minutos. Por outro lado, em um estudo 
realizado por Carreiro et al. (2017), 81,5\% dos entrevistados consideraram como bom ou ótimo o tempo de viagem até o prestador do serviço odontológico.

Segundo Castro, Oliveira e Araújo (2011), o Ministério da Saúde preconiza que o acesso deve ocorrer próximo ao local de moradia, porém, observou-se que 23,2\% dos usuários encontraram algum tipo de dificuldade para chegar à Unidade Básica de Saúde (UBS), inferindo-se que a localização da UBS pode dificultar o acesso à assistência odontológica.

Quanto às principais barreiras organizacionais encontradas, tem-se a ocorrência da falta de medicamentos, falta de materiais e deficiência na manutenção de equipamentos, aumentando, assim, o tempo e as filas de espera e a demanda reprimida (Castro, Oliveira \& Araújo, 2011; Tavares, Costa, Falcão \& Cristino, 2013), tempo de espera (Souza \& Chaves, 2010; Castro, Oliveira \& Araújo, 2011; Melo, Braga \& Forte, 2011; Chaves et al., 2012) e falta de informação (Neto, Oliveira, Zandonade \& Leal, 2012; Austregésilo, Leal, Marques, Vieira \& Alencar, 2015; Fonseca, Fonseca \& Meneghim, 2017; Solano et al., 2017).

A PNSB trouxe avanços quanto ao financiamento e expansão da saúde bucal. Porém, a dependência de tecnologias materiais pela odontologia, falta de governabilidade sobre os recursos, deficiências quanto a compra e a manutenção de equipamentos e contratação de recursos humanos são fatores que prejudicam o processo de trabalho, a assistência e, consequentemente, o acesso aos serviços odontológicos (Soares \& Paim, 2011; Tavares, Costa, Falcão \& Cristino 2013; Sanchez \& Braga, 2014).

O tempo de espera pela consulta agendada foi elevado (Melo, Braga \& Forte, 2011; Castro, Oliveira \& Araújo, 2011), variando de 15 dias (Chaves et al. 2012) a até um mês ou mais (Melo, Braga \& Forte, 2011). Souza e Chaves (2010), em estudo referente aos serviços especializados, observaram que 30\% dos usuários apontaram que o tempo de espera para a primeira consulta chegou a mais de 31 dias e 79,7\% dos usuários relataram tempo médio de até 30 dias para a consulta de retorno, chegando a alcançar até 90 dias para 15,2\% dos usuários. Outras dificuldades e barreiras organizacionais, como a existência de filas e a dificuldade para agendamento, também são relatadas pelos usuários (Castro, Oliveira \& Araújo, 2011).

A falta de informação sobre a oferta, disponibilidade e importância dos serviços odontológicos pode ser um empecilho para a sua utilização (Austregésilo, Leal, Marques, Vieira \& Alencar, 2015; Solano et al., 2017). A prevalência sobre o conhecimento das puérperas acerca de informações de interesse odontológico foi inferior a 50\% (Neto, Oliveira, Zandonade \& Leal, 2012). O estudo de Rodrigues et al. (2014) mostra uma associação significativa entre as informações preventivas dos pais sobre a saúde bucal da criança e a utilização dos serviços odontológicos, evidenciando a importância da educação em saúde. Esta associação também foi analisada pelo estudo de Paredes, Fernandes, Fernandes \& Menezes (2015) em que 51,2\% dos pais entrevistados não tinham conhecimento sobre informações preventivas aos pré-escolares e, dentre aquelas crianças que não utilizavam os serviços odontológicos, uma das justificativas dos pais foi por serem muito pequenas e não necessitarem de atendimento, constatando a necessidade de informações e educação em saúde. Solano et al. (2017) avaliaram que 54,69\% dos entrevistados em seu estudo não sabiam da existência de serviços odontológicos na Unidade de Saúde que acessavam.

A falta de informação pode ser amenizada pela sinalização interna e/ou externa nas unidades de saúde, comunicação das equipes de saúde com os usuários de forma a fortalecer o vínculo e informar sobre os serviços ofertados, acolhimento como reorganização dos serviços, atividades educativas e educação permanente em saúde (Neto, Oliveira, Zandonade \& Leal, 2012; Solano et al., 2017).

A autopercepção de saúde bucal foi o tipo de barreira cultural/subjetiva mais encontrada nos artigos analisados (Viana, Gomes, Carvalho \& Oliveira, 2010; Baldani \& Antunes, 2011; Castro, Oliveira \& Araújo, 2011; Melo, Braga \& Forte, 2011; Machado, Camargo, Jeronymo \& Bastos, 2012; Rodrigues et al., 2014; Austregésilo, Leal, Marques, Vieira \& Alencar, 2015; Emerich et al., 2015; Fonseca, Nehmy \& Mota, 2015; Oliveira, Souza, Haikal, Ferreira \& Martins, 2016; Carreiro et al., 2017; Fonseca, Fonseca \& Meneghim, 2017). A percepção de um problema de saúde pode levar à busca por atendimento 
odontológico, sendo mediada pela subjetividade de cada indivíduo e pelos valores e conhecimentos que as pessoas têm sobre a saúde e os serviços de saúde (Andersen \& Davidson, 2000).

De acordo com Machado, Camargo, Jeronymo e Bastos (2012), a variável mais fortemente associada à utilização regular dos serviços odontológicos foi a autopercepção. Indivíduos que relataram sua saúde bucal como muito boa tiveram prevalência de uso regular 4,4 vezes maior em relação àqueles que acreditavam possuir condições de saúde bucal ruim ou muito ruim. Uma pesquisa qualitativa com usuárias do SUS observou que elas percebiam que a aparência dos dentes influenciava na vida cotidiana, como na obtenção do emprego e relações sociais (Fonseca, Nehmy \& Mota, 2015).

Melo, Forte e Braga (2011) observaram que 40,3\% dos usuários de todas as idades entrevistados avaliaram sua saúde bucal como muito boa ou boa, 33,3\% consideram como regular e $26,4 \%$ se mostraram insatisfeitos. Quando se avaliou o nível de percepção somente em um grupo de adolescentes, Emerich et al. (2015) observaram que 75,9\% dos entrevistados que visitaram o dentista no último ano avaliaram sua saúde oral como boa, $21,3 \%$ como regular e apenas $2,8 \%$ como inadequada e que houve uma alta prevalência do uso dos serviços odontológicos por adolescentes. Esta autopercepção positiva da saúde bucal entre os adolescentes vai ao encontro dos resultados de Baldani e Antunes (2011) que constataram que os grupos etários dos mais jovens reportaram altos níveis de satisfação com sua saúde bucal e menos necessidade de tratamento odontológico.

Por conseguinte, a percepção dos indivíduos quanto às necessidades de atendimento odontológico constitui um notável indicador na determinação da utilização dos serviços, podendo ser alcançado, também, por ações de educação em saúde (Castro, Oliveira \& Araújo, 2011).

Cabe ainda registrar que as contribuições dos artigos selecionados para esta revisão integrativa apoiaram-se, em sua grande maioria, na abordagem epidemiológica e quantitativa do estudo sobre o acesso e utilização dos serviços odontológicos. Neste sentido, reflexões teóricas e conceituais, apontam para as contribuições das abordagens qualitativas sobre o tema e potencialidades de outras perspectivas teóricas, a exemplo da sociologia de Bourdieu (Cruz, Chaves \& Cangussu, 2016).

\section{Considerações Finais}

A análise dos artigos selecionados traz informações sobre as principais barreiras de acesso aos serviços de saúde bucal no SUS, como as barreiras geográficas, organizacionais e culturais/pessoais.

Considera-se, porém, algumas limitações da pesquisa, como o não cruzamento entre os descritores e o uso de duas bases de dados, com a inclusão de artigos sem a incorporação de dissertações ou teses. Já em relação às contribuições, a sistematização dos achados trazendo as estratégias de superação das dificuldades de acesso pode contribuir para o planejamento de ações e intervenções no sistema de saúde de forma a atenuá-las.

Apesar dos avanços e melhorias de acesso aos serviços odontológicos pela PNSB, observa-se a necessidade de reorganização dos modelos e práticas vigentes no sentido de minimizar as barreiras de acesso, sobretudo através de ações que alcancem as populações mais vulneráveis como forma de justiça e equidade social. Espera-se que este estudo possa fomentar debates acerca do enfrentamento das barreiras de acesso que dificultam o uso dos serviços odontológicos.

Ademais, sugere-se que trabalhos futuros possam contemplar outros grupos sociais em estado de vulnerabilidade, a exemplo de homens em condições socioeconômicas desfavoráveis, população LGBTQIA+, população negra, comunidades indígenas, ribeirinhas, dentre outros não mencionados nos artigos selecionados, de forma a ampliar as discussões em torno de estratégias que permitam a inclusão e facilidade de acesso aos serviços de saúde bucal por estes grupos, efetivando o alcance universal do SUS e diminuindo as iniquidades sociais. 
Research, Society and Development, v. 10, n. 3, e17610313194, 2021

(CC BY 4.0) | ISSN 2525-3409 | DOI: http://dx.doi.org/10.33448/rsd-v10i3.13194

\section{Referências}

Abreu-de-Jesus, W. L., \& Assis, M. M. A. (2010). Revisão sistemática sobre o conceito de acesso nos serviços de saúde: contribuições do planejamento. Ciência e Saúde Coletiva, 15(1):161-70. http://dx.doi.org/10.1590/S1413-81232010000100022

Andersen, R. M. (1995). Revisiting the behavioral model and Access to medical care: does it matter? Journalof Health and Social Behavior, 36(1), 1-10. https://pubmed.ncbi.nlm.nih.gov/7738325/

Andersen, R. M. \& Davidson, P. L. (2000). Improving Access to care in America: individual and contextual indicators. In: Andersen R. M., et al. Changing the U.S. healthcare system: key issues in health services policy and management. (3rd ed.), Jossey-Bass.

Austregésilo, S. C., Leal, M. C. C., Marques, A. P. O., Vieira, J. C. M., \& Alencar, D. L. (2015). Acessibilidade a serviços de saúde bucal por pessoas idosas: uma revisão integrativa. Revista Brasileira de Geriatria e Gerontologia, 18(1), 189-199. https://doi.org/10.1590/1809-9823.2015.13179

Baldani, M. H., \& Antunes, J. L. (2011). Inequalities in access and utilization of dental services: a cross-sectional study in an area covered by the Family Health Strategy. Caderno de Saúde Pública, 27(Sup 2), 5272-5283. https://doi.org/10.1590/S0102-311X2011001400014

Barros, S. G., Medina, M. G., Carcereri, D. L., Ruiz, D.C., \& Peres, A. C. O. (2016). Atenção primária e saúde bucal. In Chaves S. C. Política de Saúde Bucal no Brasil. Editora EDUFBA.

Brasil. (2000). Ministério da Saúde. Portaria nº 1.444 GM/MS de 28 de dezembro de 2000. Estabelece incentivo financeiro para a reorganização da atenção à saúde bucal prestadas nos municípios por meio do programa saúde da família. Brasília.

Brasil. (2004). Ministério da Saúde. Departamento de Atenção Básica. Diretrizes da Política Nacional de Saúde Bucal.

Brasil. (2006). Política Nacional de Atenção Básica. Ministério da Saúde.

Brasil. (2011). SB Brasil 2010: Pesquisa Nacional de Saúde Bucal. Resultados principais. Ministério da Justiça. Secretaria de Atenção à Saúde. Secretaria de Vigilância em Saúde. Departamento de Atenção à Saúde. Coordenação Geral de Saúde Bucal, Brasília.

Carreiro, D. L. et al. (2017). Uso de serviços odontológicos de forma regular na população de Montes Claros, MG, Brasil. Ciência \& Saúde Coletiva, 22(12), 4135-4150. https://doi.org/10.1590/1413-812320172212.04492016

Castro, R. D., Oliveira, A. G., \& Araújo, I. M. (2011). Estudo da Acessibilidade Organizacional aos Serviços de Saúde Bucal de um Município de Pequeno Porte do Nordeste brasileiro. Revista Brasileira de Ciências da Saúde, 14(4), 65-78. https://periodicos.ufpb.br/index.php/rbcs/article/view/9719/5688

Cavalcanti, R. P., Gaspar, G. S., \& Goes, P. S. (2012). Utilização e Acesso aos Serviços de Saúde Bucal do SUS -Uma Comparação entre Populações Rurais e Urbanas. Pesquisa Brasileira em Odontopediatria Clínica Integrada, 12(1), 121-126. 10.4034/PBOCI.2012.121.19

Chaves, S. C. et al. (2012). Características do acesso e utilização de serviços odontológicos em municípios de médio porte. Ciência \& Saúde Coletiva, 17(11), 3115-3124. https://doi.org/10.1590/S1413-81232012001100027

Cruz, D. N., Chaves, S., \& Cangussu, M. C. (2016). A utilização dos serviços odontológicos: elementos teóricos e conceituais. in Chaves, S. C. Política de Saúde Bucal no Brasil. Editora EDUFBA.

Donabedian A. (1973). Aspects of medical care administration. Harvard University Press.

Donabedian, A. (2003). An introduction to quality assurance in healthcare. Oxford University.

Emerich et al. (2015). Access to dental services and related factors in adolescents from Vitória, Espírito Santo, Brazil, 2011. Brazilian Research in Pediatric Dentistry and Integrated Clinic, 15(1), 253-262. http://dx.doi.org/10.4034/PBOCI.2015.151.27

Esposti, C. D., Cavaca, A. G., Côco, L. S. A., Santos-Neto, E. T., \& Oliveira, A. E. (2016). As dimensões do acesso aos serviços de saúde bucal na mídia impressa. Saúde e Sociedade. São Paulo, 25(1), 19-30. https://doi.org/10.1590/S0104-12902016141706

Fonseca, L. L., Nehmy, R. M., \& Mota, J. A. (2015). O valor social dos dentes e o acesso aos serviços odontológicos. Ciência \& Saúde Coletiva, 20(10), 31293138. DOI: 10.1590/1413-812320152010.00172015

Fonseca, E. P., Fonseca, S. G., \& Meneghim, M. C. (2017). Análise do acesso aos serviços odontológicos públicos no Brasil. ABCS Health Sciences, 42(2), 85-92. https://doi.org/10.7322/abcshs.v42i2.1008

Hobdell, M. H. (2007). Poverty, oral health and human development: contemporary issues affecting the provision of primary oral healthcare. Journal of the American Dental Association, 138(11), 1433-1436. 10.14219/jada.archive.2007.0076

Machado, L. P., Camargo, M. B. J., Jeronymo, J. C. M., \& Bastos, G. A. N. (2012). Uso regular de serviços odontológicos entre adultos e idosos em região vulnerável no sul do Brasil. Revista de Saúde Pública, 46(3), 526-33. https://doi.org/10.1590/S0034-89102012000300015

Mattos, G. C. M., Ferreira, E. F., Leite, I. C. G., \& Greco, R. M. (2014). A inclusão da equipe de saúde bucal na Estratégia Saúde da Família: entraves, avanços e desafios. Ciência e Saúde Coletiva, 19(2), 373-382. 10.1590/1413-81232014192.21652012

Melo, A. C., Braga, C. C., \& Forte, F. D. (2011). Acessibilidade ao Serviço de Saúde Bucal na Atenção Básica: Desvelando o Absenteísmo em uma Unidade de Saúde da Família de João Pessoa -PB. Revista Brasileira de Ciências da Saúde, 15(3), 309-318. https://periodicos.ufpb.br/ojs2/index.php/rbcs/article/view/10734.

Mendes, K. D. S., Silveira, R. C. C. P., \& Galvão, C. M. (2008). Revisão integrativa: método de pesquisa para a incorporação de evidências na saúde e na enfermagem. Texto \& Contexto Enfermagem, 17(4), 758-64. http://dx.doi.org/10.1590/S0104-07072008000400018. 
Research, Society and Development, v. 10, n. 3, e17610313194, 2021

(CC BY 4.0) | ISSN 2525-3409 | DOI: http://dx.doi.org/10.33448/rsd-v10i3.13194

Narvai, P. C. (2006). Saúde bucal coletiva: caminhos da odontologia sanitária à bucalidade. Revista de Saúde Pública, 40, 141-7. https://www.scielo.br/pdf/rsp/v40nspe/30633

Neto, E. T. S., Oliveira, A. E., Zandonade, E., \& Leal, M.C. (2012). Acesso à assistência odontológica no acompanhamento pré-natal. Ciência \& Saúde Coletiva, 17(11), 3057-3068. https://doi.org/10.1590/S1413-81232012001100022.

Oliveira, R. F. R., Souza, J. G. S., Haikal, D. S., Ferreira, E. F., \& Martins, A. M. E. (2016). Equidade no uso de serviços odontológicos provenientes do SUS entre idosos: estudo de base populacional. Ciência \& Saúde Coletiva. 21(11), 3509-3523. https://doi.org/10.1590/1413-812320152111.22532015

Paim, S. J. (2016). O que é o SUS. Editora Fiocruz.

Paredes, S. O., Fernandes, J. R. L., Fernandes, J. M. F. A., \& Menezes, V. A. (2015). Utilização dos serviços odontológicos por pré-escolares em um município de pequeno porte do Estado da Paraíba. Revista de Odontologia da UNESP, 44(3), 181-187. https://doi.org/10.1590/1807-2577.1069

Peres, K. G. et al. (2012). Redução das desigualdades sociais na utilização de serviços odontológicos no Brasil entre 1998 e 2008 . Revista de Saúde Pública, 46(2), 250-258. https://doi.org/10.1590/S0034-89102012000200007

Petersen, P. E., Bourgeois, D., Ogawa, H., Estupinan-Day, O., \& Ndiaye, C. (2005). The global burdenof oral diseases and risks to oralhealth. Bulletin of the World Health Organization, 83(9). https://www.scielosp.org/pdf/bwho/2005.v83n9/661-669/

Pinto, V. P. et al. (2014). Avaliação da acessibilidade ao Centro de Especialidades Odontológicas de abrangência macrorregional de Sobral, Ceará, Brasil. Ciência \& Saúde Coletiva, 19(7), 2235-2244. https://doi.org/10.1590/1413-81232014197.09862013

Rocha-Buelvas, A. (2013). Análisis sobre elacceso a losservicios de lasalud bucal: um indicador de equidad. Revista Gerencia y Políticas de Salud, 12(25), 96112. http://www.scielo.org.co/pdf/rgps/v12n25/v12n25a08.pdf

Rodrigues, L. A. et al. (2014). Uso de serviços odontológicos entre pré-escolares: estudo de base populacional. Ciência \& Saúde Coletiva, 19(10), 4247-4256. https://doi.org/10.1590/1413-812320141910.13382013

Sanchez, H. F., \& Braga, S. C. (2014). Melhoria do acesso à saúde bucal na Estratégia de Saúde da Família: uma revisão de literatura. Revista Atenção Primária à Saúde, 17(3), 397-402. https://periodicos.ufjf.br/index.php/aps/article/view/15466.

Santos, G. L., Freitas, V. S., Andrade, M. C., \& Oliveira, M. C. (2010). Fumo e álcool como fatores de risco para o câncer bucal. Odontol. Clín.-Cient., 9(2), 131-133. http://revodonto.bvsalud.org/pdf/occ/v9n2/a08v9n2.pdf.

Soares, F. F., Chaves, S. C., \& Cangussu, M. C. (2013). Desigualdade na utilização de serviços de saúde bucal na atenção básica e fatores associados em dois municípios brasileiros. Revista Panamericana de Salud Pública, 34(6). https://pesquisa.bvsalud.org/portal/resource/pt/lil-702714.

Soares, C. L. M., \& Paim, J. S. (2011). Aspectos críticos para a implementação da política de saúde bucal no município de Salvador, Bahia, Brasil. Caderno de Saúde Pública. 27(5), 966-74. https://www.readcube.com/articles/10.1590/s0102-311x2011000500014.

Solano et al. (2017). Utilização de serviços de saúde bucal na atenção primária: perspectivas dos usuários do SUS de um município do sul do Brasil. Revista Ciência Plural, 3(3), 81-92. https://pesquisa.bvsalud.org/portal/resource/pt/biblio-883518.

Souza, L. F., \& Chaves, S. C. (2010). Política nacional de saúde bucal: acessibilidade e utilização de serviços odontológicos especializados em um município de médio porte na Bahia. Revista Baiana Saúde Pública, 34(2), 371-387. http://files.bvs.br/upload/S/0100-0233/2010/v34n2/a1814.pdf.

Tavares, R. P., Costa, G. C., Falcão, M. L. M., \& Cristino, P. S. (2013). A organização do acesso aos serviços de saúde bucal na estratégia de saúde da família de um município da Bahia. Saúde em Debate, 37(99), 628-635. https://doi.org/10.1590/S0103-11042013000400010.

Travassos, C. \& Martins, M. (2004). Uma revisão sobre os conceitos de acesso e utilização de serviços de saúde. Caderno de Saúde Pública, 20 (Sup 2), 190198. https://doi.org/10.1590/S0102-311X2004000800014

Viana, A. M., Gomes, M. J., Carvalho, R. B., \& Oliveira, E. R. A. (2010). Acessibilidade dos idosos brasileiros aos serviços odontológicos. Revista da Faculdade de Odontologia, 15(3), 319-324. http://revodonto.bvsalud.org/scielo.php?pid=S1413-40122010000300019\&script=sci_arttext.

Viegas, A. P., Carmo, R. F., \& Luz, Z. M. (2015). Fatores que influenciam o acesso aos serviços de saúde na visão de profissionais e usuários de uma unidade básica de referência. Saúde e Sociedade, 24(1), 100-112. https://doi.org/10.1590/S0104-12902015000100008 